\title{
Analysis of blood clot degradation fragment sizes in relation to plasma flow velocity
}

\author{
Franci Bajd ${ }^{1}$, Jernej Vidmar ${ }^{1,2}$, Aleš Blinc ${ }^{3}$ and Igor Serša ${ }^{1,4}$ \\ ${ }^{1}$ Jožef Stefan Institute, Ljubljana 1000, Slovenia \\ ${ }^{2}$ Institute of Physiology, Medical faculty of Ljubljana, Ljubljana 1000, Slovenia \\ ${ }^{3}$ Department of Vascular Diseases, University of Ljubljana Medical Centre, Ljubljana 1000, Slovenia \\ ${ }^{4}$ EN-FIST Centre of Excellence, Ljubljana 1000, Slovenia
}

\begin{abstract}
Thrombolytic therapy aims to dissolve blood clots and restore vessel patency. Our hypothesis is that the therapy depends not only on chemical reactions of the fibrinolytic system, but also on mechanical forces exerted by streaming blood on the clot surface. The aim of the study was to analyze the role of mechanical forces in promoting thrombolysis and their relation to the maximum size of removed clot fragments. Non-occlusive whole-blood clots were exposed to flow of plasma containing the thrombolytic agent rt-PA. Plasma, perfusing through the clot, was collected and the sizes of clot degradation fragments in the plasma were analyzed by optical microscopy. Theoretical models for the maximum clot fragment size as a function of blood flow velocity were developed based on the relation between surface or volume clot binding forces and the opposing forces of the streaming plasma in the laminar or turbulent flow regime. The best agreement between experimental results and models was obtained for the volume binding forces and the laminar flow model, in which the maximum clot fragment size was linearly dependent on the plasma flow velocity. Such result could not be obtained if thrombolysis would be purely a biochemical process. Therefore, the result confirms our hypothesis that thrombolysis is also strongly influenced by the mechanical forces of streaming plasma.
\end{abstract}

Key words: Blood clot — Degradation size distribution — Optical microscopy — Thrombolysis

Abbreviations: RBC, red blood cell; Re, Reynolds number; rt-PA, recombinant tissue plasminogen activator.

\section{Introduction}

The goal of thrombolytic therapy (thrombolysis) is efficient dissolution of obstructing thrombi and restoration of vessel patency. Thrombolysis is used in treatment of ischemic stroke (White-Bateman et al. 2007), pulmonary embolism (Kucher and Goldhaber 2006) and in acute arterial thrombosis (Kandarpa 1999). The biochemical basis of thrombolysis is enzymatic proteolysis of the fibrin network that entraps blood cells (Collen and Lijnen 1995). Fibrinolysis is triggered by activation of the proenzyme plasminogen

Correspondence to: Igor Serša, Jožef Stefan Institute, Jamova 39, 1000 Ljubljana, Slovenia

E-mail: igor.sersa@ijs.si into the active serine protease plasmin by plasminogen activators, e.g., recombinant tissue plasminogen activator (rt-PA) (Blinc and Francis 1996). Plasmin acts locally on the fibrin polymer, whereas in circulating plasma it is rapidly inactivated by its specific inhibitor antiplasmin (Collen et al. 1995).

Several experiments on dissolution of non-occlusive blood clots in model perfusion systems as well as clinical results indicate that the rate of thrombolysis depends significantly on the blood flow streaming axially along the clot (Blinc et al. 1996; Sakharov and Rijken 2000; Tratar et al. 2004). The effects of blood flow cannot be attributed only to different permeation rates of the thrombolytic agent into clots, but also to differences in shear forces of flowing blood exerted to the clot surface (Sersa et al. 2007). 
We have proposed earlier that larger mechanical forces of rapidly streaming plasma remove larger and only partially chemically degraded clot fragments from the clot surface, in contrast to lower forces of slowly flowing plasma (Sersa et al. 2007). However, analysis of fibrin degradation fragments by gel electrophoresis (Marder and Francis 1983) in relation to the axial plasma flow velocity did not prove suitable, since the abundance of plasma proteins dwarfed minute quantities of fibrin degradation fragments and prevented their detection by biochemical methods. Therefore, we decided to look for clot degradation fragments not on the macromolecular (nanometer scale), but rather on the micrometer scale, looking at cell clusters still bound together by the partly degraded fibrin network.

The aim of the study was to analyze the role of mechanical forces in promoting thrombolysis and their relation to the maximum size of clot fragments removed from the clot surface (maximum clot fragment size) during thrombolysis. A theoretical model that explains how forces of streaming blood plasma promote clot degradation was developed. The model yields predictions for the maximum clot fragment size as a function of blood flow velocity for different flow regimes and clot binding forces. The predictions were verified by optical microscopy that can reliably detect clot degradation fragment sizes as a function of the plasma flow velocity.

\section{Theory}

Our model of thrombolysis is based on the assumption that the shear forces of streaming blood promote clot dissolution because regions of the incompletely chemically degraded clot, i.e., red cell clusters still bound together by partially degraded fibrin polymers, are removed from the clot surface. This contribution to clot degradation may be considered as mechanical thrombolysis. The model assumes that the rate of mechanical thrombolysis is mainly governed by either viscous or turbulent mechanical forces at the blood-clot interface. The fragments are removed earlier and their sizes are bigger if the flow is fast, while in the slow flow regime or in the absence of flow it is expected that the fragments would be removed later and their sizes would be smaller due to the pronounced effect of chemical degradation.

Two flow regimes may be considered: a laminar and turbulent flow regime. In addition, the binding force between partially degraded clot fragments may be considered proportional to the fragment surface or alternatively to its volume, if the clot is considered homogeneous and nonretracted. Let us consider what force is needed to remove a cubic fragment with a side of length $a$. The fragment surface area is $6 a^{2}$ and its volume $a^{3}$ which results in the following binding forces

$$
F_{b}= \begin{cases}k_{S} 6 a^{2} & ; \text { binding proportional to surface } \\ k_{V} a^{3} & ; \text { binding proportional to volume }\end{cases}
$$

Here $k_{S}$ and $k_{V}$ are two proportionality constants, for surface and for volume binding, respectively. If the flow is laminar then the force of streaming blood on the exposed surface of the clot is of a viscous nature and is therefore proportional to the blood velocity $v$, i.e., to the flow rate. However, if the flow regime is turbulent, then the force of streaming blood on the exposed surface of the clot is proportional to the blood velocity squared $v^{2}$, i.e., to the flow rate squared

$$
F=\left\{\begin{array}{cc}
f_{L} a^{2} v ; & \text { laminar flow } \\
f_{T} a^{2} v^{2} ; & \text { turbulent flow }
\end{array}\right.
$$

where $f_{L}$ and $f_{T}$ are two proportionality constants, for laminar and for turbulent flow, respectively. The constants $f_{L}$ and $f_{T}$ include all flow specific characteristics except velocity. The fragment is removed from the clot surface when the mechanical force $F$ of streaming blood on the fragment is greater than the force $F_{b}$ that binds the fragment to the remaining clot, i.e., $F>F_{b}$. This results in the following conditions for removal of the fragment from the clot surface. For the binding force, that is proportional to the surface of the fragment to be removed, a limiting blood velocity exists, below which mechanical thrombolysis is non-existent, i.e., fragments cannot be mechanically removed from the surface of the clot. Mechanical thrombolysis occurs when

$$
v \geq\left\{\begin{array}{ccl}
6 k_{S} / f_{L} & ; & \text { laminar flow } \\
\sqrt{6 k_{S} / f_{T}} & ; & \text { turbulent flow }
\end{array}\right.
$$

In principle, the fragment size in that case does not depend on the blood velocity if the flow velocity exceeds the limiting value; i.e., clot fragments are distributed up to sizes of $a_{\max }$ (maximum clot fragment size) that has no upper limit. However, if the binding force is proportional to the fragment volume this is no longer true. In that case clot fragments are limited in size by the velocity dependent maximum clot fragment size

$$
a_{\max }=\left\{\begin{array}{ccl}
\left(f_{L} / k_{V}\right) v & ; & \text { laminar flow } \\
\left(f_{T} / k_{V}\right) v^{2} & ; & \text { turbulent flow }
\end{array}\right.
$$

Since the model describes mechanical thrombolysis of a heterogeneous structure predominantly composed of red blood cells (RBCs), sizes of the removed clot fragments can- 
not be reduced down to zero as this would imply mechanical disintegration of individual RBCs. This cannot be induced by physiological hemodynamic conditions (Dao et al. 2003). Therefore, clot fragment sizes have in addition to the upper limit of $a_{\max }$ also the lower limit that corresponds to the size of a single $\mathrm{RBC}\left(a_{R B C}\right)$

$$
a_{R B C} \leq a \leq a_{\max }
$$

Figure 1 depicts theoretically predicted regimes of a clot fragment mechanical removal from the exposed clot surface (mechanical thrombolysis). Reference velocities in graphs in Fig. 1 correspond to $v_{S L}=6 k_{S} / f_{L}$ (surface-binding forces, laminar flow), $v_{S T}=\left(6 k_{S} / f_{T}\right)^{1 / 2}$ (surface-binding forces, turbulent flow), $v_{V L}=k_{V} a_{R B C} / f_{L}$ (volume-binding forces, laminar flow) and to $v_{V T}=\left(k_{V} a_{R B C} / f_{T}\right)^{1 / 2}$ (volume-binding forces, turbulent flow). The first two reference velocities are defined in Eq. (3), while the last two reference velocities were obtained by normalization of Eq. (4) to $a_{R B C}$.

The criterion to discriminate between laminar and turbulent flow regimes is defined by Reynolds number $\operatorname{Re}=d \rho v / \eta$, where $d$ is the diameter of a perfusion channel, $\rho$ is blood plasma density, $v$ is an average plasma flow velocity through the perfusion channel and $\eta$ is viscosity of blood plasma (Landau and Lifshitz 1995). For normal, smooth surfaces Re was experimentally found to be less than 2000 in the laminar flow regime and more than 3000 in the turbulent flow regime (Landau et al. 1995). Taking into account the blood density of $1035 \mathrm{~kg} / \mathrm{m}^{3}$ and the blood viscosity of $16 \cdot 10^{-4} \mathrm{~Pa} \cdot \mathrm{s}$ (Zidanšek et al. 1995), the average velocity in the perfusion channel through or along the non-occlusive clot and the characteristic dimensions of this channel, Re can be a good measure to distinguish between laminar and turbulent flow regimes.
A
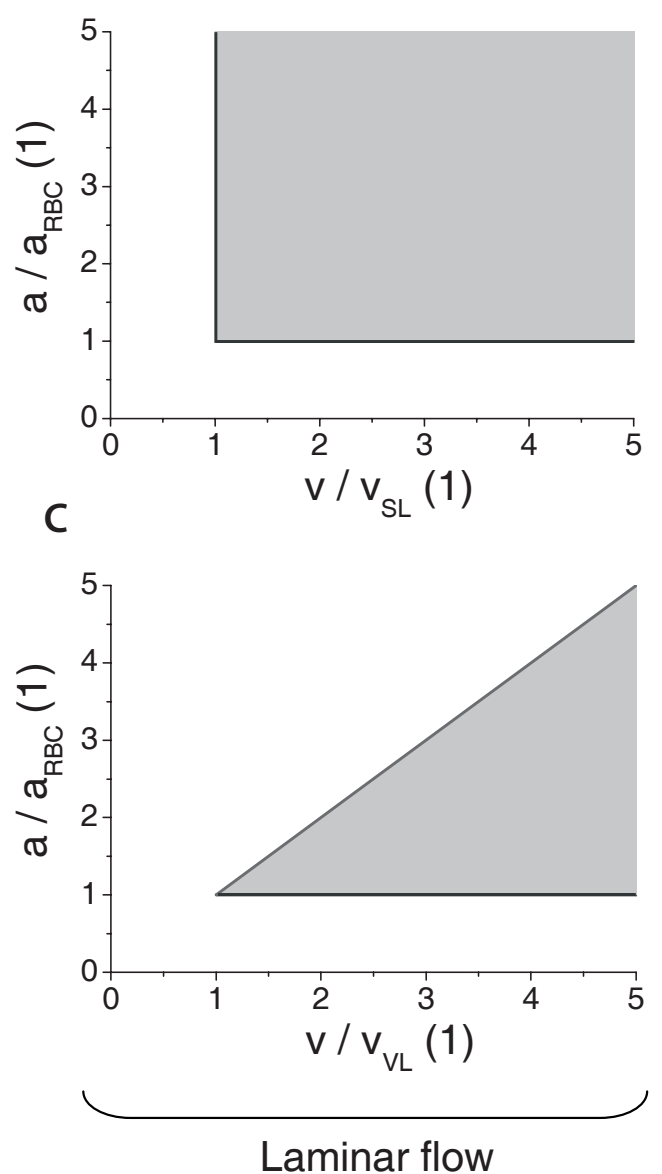

B
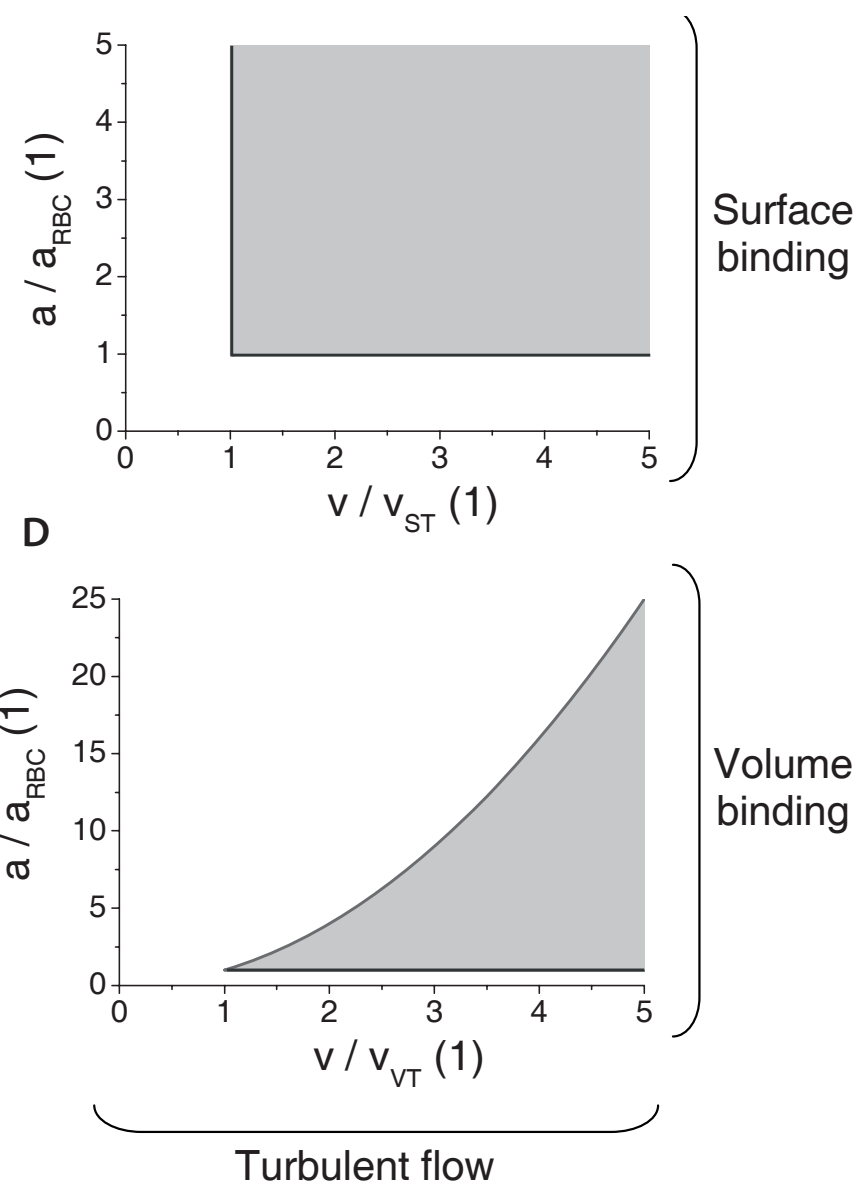

Figure 1. Theoretical prediction for sizes of removed clot fragments (shaded areas) for surface-force fragment binding (A, B) and for volume-force fragment binding $(\mathrm{C}, \mathrm{D})$ in a laminar flow regime $(\mathrm{A}, \mathrm{C})$ and in a turbulent flow regime (B, D). Clot fragments cannot be smaller in size than $a_{R B C}$ (size of a single RBC) and cannot exceed $a_{\max }$ (maximum clot fragment size), which is unlimited in the case of surface binding and is velocity-dependent in the case of volume binding. 


\section{Materials and Methods}

\section{Blood clots and artificial perfusion system}

Sizes of blood clot degradation fragments were measured in model thrombolytic experiments. The experiments were performed on artificial non-occlusive and non-retracted whole blood clots that were inserted into an artificial perfusion system consisting of a peristaltic pump (913 Mityflex), a plasma reservoir, plastic hoses and a $3 \mathrm{~mm}$ diameter glass tube with the clot (Fig. 2). Blood clots were made of blood collected from the cubital veins of healthy volunteers. Blood was drawn into tubes (Vacuntainer, Becton-Dickinson, Germany) containing $1 \mathrm{ml}$ of $0.129 \mathrm{~mol} / \mathrm{l} \mathrm{Na}$-citrate. To neutralize the anticoagulation effect of $\mathrm{Na}$-citrate, $50 \mu \mathrm{l}$ of $\mathrm{CaCl}_{2}$ at a concentration of $2 \mathrm{~mol} / \mathrm{l}$ was added to blood and then clotting was initiated by adding thrombin (Thrombin, Sigma, Germany) at a final concentration of $0.44 \mu \mathrm{g} / \mathrm{ml}$. Spontaneous retraction of blood clots was inhibited by the phosphodiesterase inhibitor UDCG 212 (Boehringer, Germany) at a concentration of $20 \mu \mathrm{l} / \mathrm{ml}$ (Šabovič et al. 1990). Before coagulation, blood was injected into $3 \mathrm{~cm}$ long cylindrical glass tubes of $3 \mathrm{~mm}$ diameter, where blood clots were formed. Glass tubes had their inner surface etched in order to ensure good adherence of the clots. The distal end of the glass tube was covered by a plastic mesh with $1 \mathrm{~mm}$ openings to prevent clot embolization during thrombolysis. After approximately one hour, when the fibrin mesh completely polymerized (Grobelnik et al. 2008), the clots were pierced lengthways along the glass-clot interface by a $0.7 \mathrm{~mm}$ diameter needle to create a flow channel along the clot. The proximal end of the tube was connected through a $0.6 \mathrm{~m}$ long flexible hose of $3 \mathrm{~mm}$ diameter to the peristaltic pump. The pump was filled from a 0.21 reservoir of human plasma mixed with rt-PA (Sigma Aldrich) to a final concentration of $20 \mu \mathrm{g} / \mathrm{ml}$. Plasma in the reservoir was heated to normal body temperature $\left(37^{\circ} \mathrm{C}\right)$ to mimic physiological conditions. In the experiments we used recently outdated human plasma provided by the $\mathrm{Na}$ tional Blood Transfusion Centre. The hoses of the perfusion system as well as the glass tube with the clot were positioned horizontally. Before thrombolytic experiments, flow regimes of the pump were calibrated by measuring plasma volume flow rates without clots in the perfusion system.

\section{Conditions of thrombolysis}

The following protocol was used during model thrombolytic experiments. First, to initiate biochemical thrombolysis and to remove possible clot debris in the flow channel resulting from piercing the clot or remaining air bubbles in the perfusion system, blood clots were permeated with rt-PAcontaining plasma for $3 \mathrm{~min}$ in the slow flow regime with a volume flow of $4 \mathrm{ml} / \mathrm{min}(v=0.18 \mathrm{~m} / \mathrm{s})$. After the first 3 minutes, the flow regime remained unchanged or the volume flow rate was increased to either $17 \mathrm{ml} / \mathrm{min}(v=0.75 \mathrm{~m} / \mathrm{s})$ or $34 \mathrm{ml} / \mathrm{min}(v=1.5 \mathrm{~m} / \mathrm{s})$. Dissolution of at least three clots was measured in each of the three plasma flow regimes. Approximately $5 \mathrm{~min}$ after the flow rate increase (or no flow rate change in the slowest flow regime) $1 \mathrm{ml}$ of plasma leaving

A
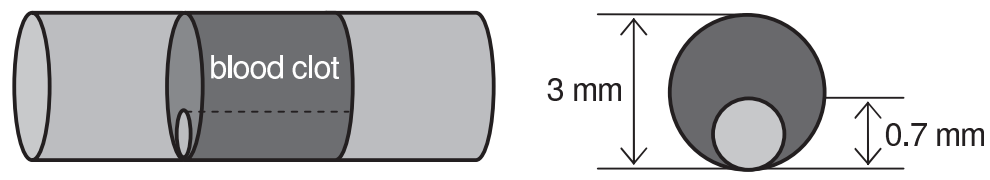

$\longleftrightarrow$

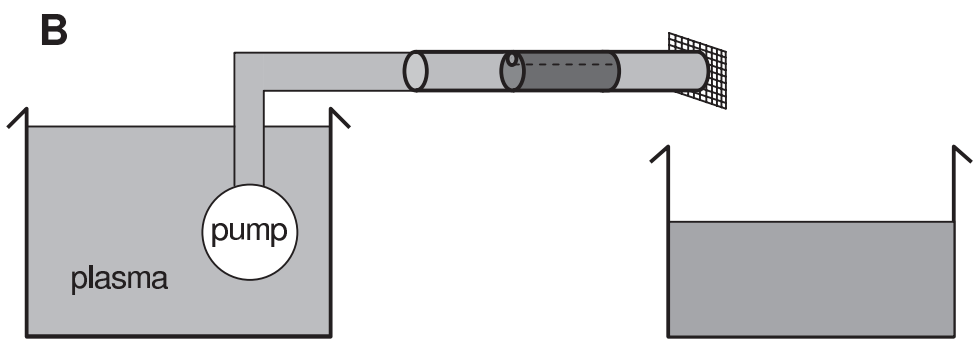

Figure 2. Schematic representation of the experimental setup. Non-occlusive model whole-blood clots were formed in glass tubes (A) that were connected by flexible hoses to a perfusion system with a peristaltic pump (B). 
the flow channel along the clot was collected in a vial that contained $25 \mu \mathrm{l}$ of $\varepsilon$-aminocaproic acid at a concentration of $0.02 \mathrm{~mol} / \mathrm{l}$ (Sigma Aldrich) to prevent further enzymatic fibrinolysis. A small drop $(20 \mu \mathrm{l})$ of plasma containing clot degradation fragments was then placed on a microscope glass slide and gently covered with a cover slip. Several drops (from five to ten) of each vial were analyzed.

\section{Optical microscopy}

Optical microscopy of the plasma sample was done immediately after the sample collection on a Nikon 80i Eclipse microscope, equipped with the Nikon Plan Fluor 20× objective with a numerical aperture of 0.5 . The microscope was equipped with an auto-focus feature, a motorized computercontrolled microscope stage and a digital camera enabling acquisition of JPEG or TIF digital images on a matrix of 1920 $\times 2520$ pixels, which corresponded to a field of view (FOV) of $324 \mu \mathrm{m} \times 434 \mu \mathrm{m}$. To randomize the assessment of clot degradation fragment sizes, sample images were taken in positions of a $5 \times 5$ matrix with a $1 \mathrm{~mm}$ gap.

\section{Image analysis}

The images were analyzed using the NIS Elements (Nikon) fragment tracking software to measure clot fragment sizes. Regions of interest (ROI) for fragment tracking software analysis were either thresholded automatically or defined manually in the case of a low signal-to-noise ratio to discriminate single RBCs and RBC clusters from background plasma. Additional image processing was done by the ImageJ computer program (Java version of Image, NIH USA). The output of the fragment tracking software was a distribution of the clot degradation fragment count as a function of the clot degradation fragment area $S$. The distributions were multiplied by the corresponding bin area $S_{\text {ind }}$ and normalized to obtain normalized fragment area distributions, i.e., the proportion of different fragment sizes per total area of all fragments in a given flow regime. The normalized fragment area distributions were then fitted with the power-law function

$$
G(S)=A+B\left(S / S_{R B C}\right)^{-C}
$$

which was found adequate to describe mechanical fragmentation of various materials (Kadono 1997; Timar et al. 2010). Here, parameters $A, B$, and $C$ control shape of the power-law function and $S_{R B C}=50 \mu \mathrm{m}^{2}$ corresponds to a surface area of a single RBC. As individual microscopy images did not contain enough data, at least 15 images in each of the flow regimes were combined to obtain the distribution of clot degradation fragment sizes. The data were analyzed and the corresponding graphs of clot fragment size analyses were plotted with the Origin computer program (OriginLab, USA).
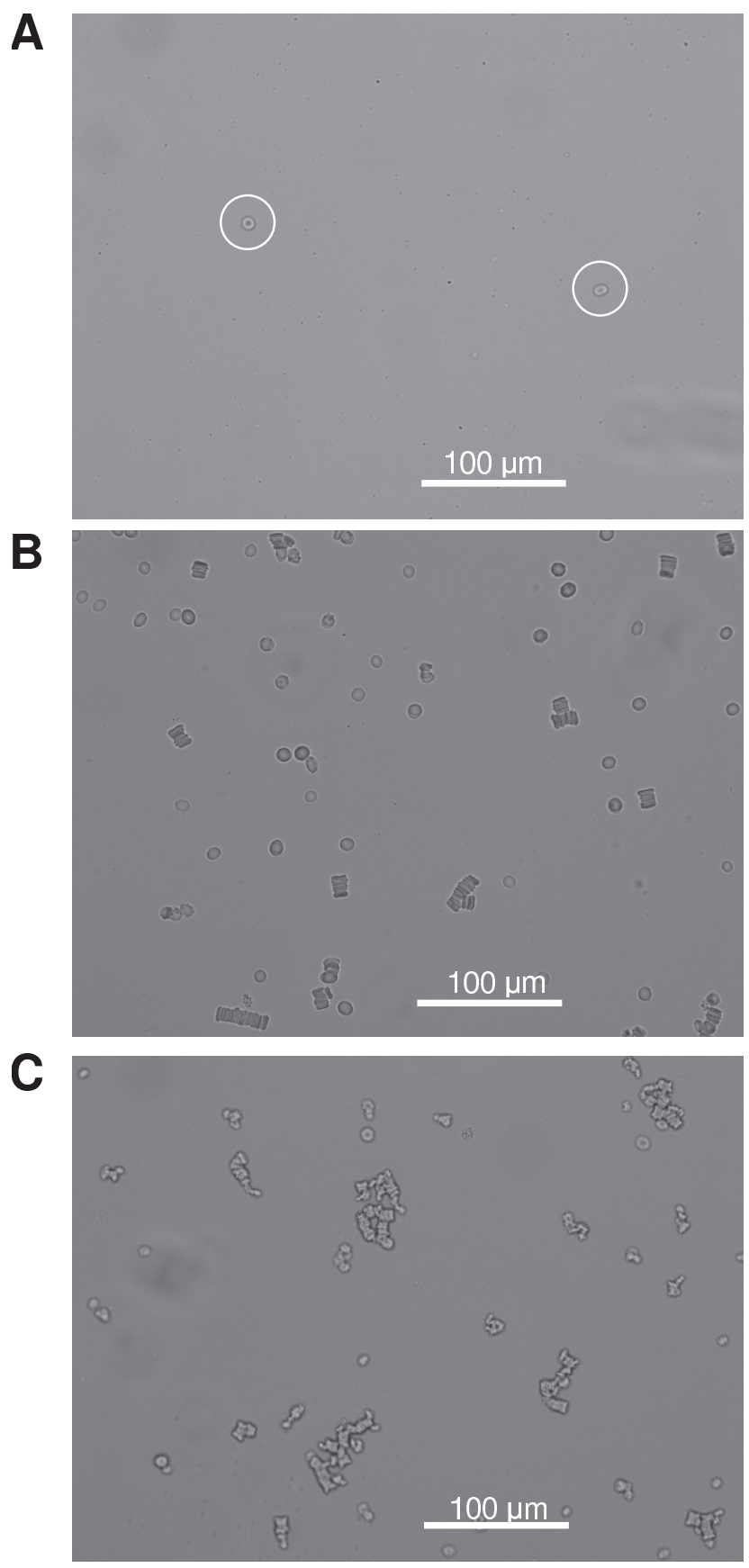

Figure 3. Optical-microscopy images of plasma containing clot degradation fragments for three different flow regimes with perfusion channel plasma velocities: slow flow, $v=0.18 \mathrm{~m} / \mathrm{s}(\mathbf{A})$; medium flow, $v=0.75 \mathrm{~m} / \mathrm{s}(\mathbf{B})$; fast flow, $v=1.5 \mathrm{~m} / \mathrm{s}(\mathbf{C})$.

\section{Results}

Representative optical microscopy images of plasma samples acquired during thrombolysis in the three different flow regimes are shown in Fig. 3. Average velocities in the flow 
channel were $0.18 \mathrm{~m} / \mathrm{s}$ in the slow flow regime, $0.75 \mathrm{~m} / \mathrm{s}$ in the medium flow regime and $1.5 \mathrm{~m} / \mathrm{s}$ in the fast flow regime. Differences between the images in Fig. 3 are apparent. They not only differ in the number of visible degradation fragments, but also in their sizes. Degradation fragments were in the range from single RBCs to larger RBC clusters in which cells were bound together by a partially chemically degraded

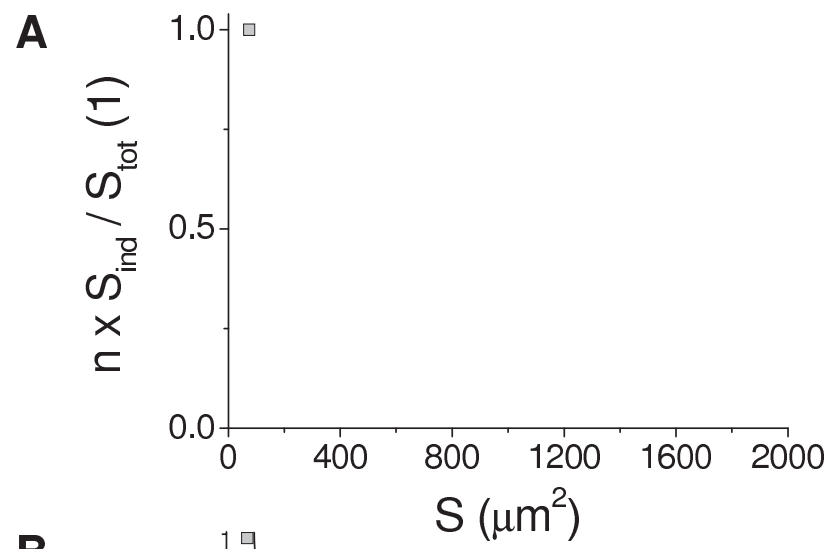

B

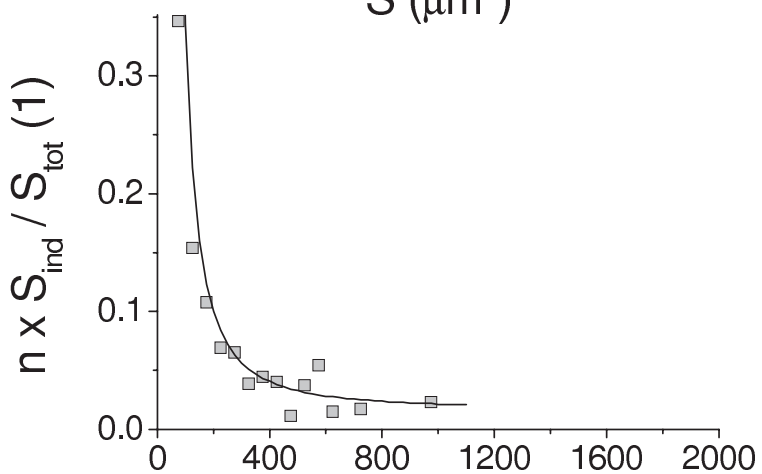

\section{C}

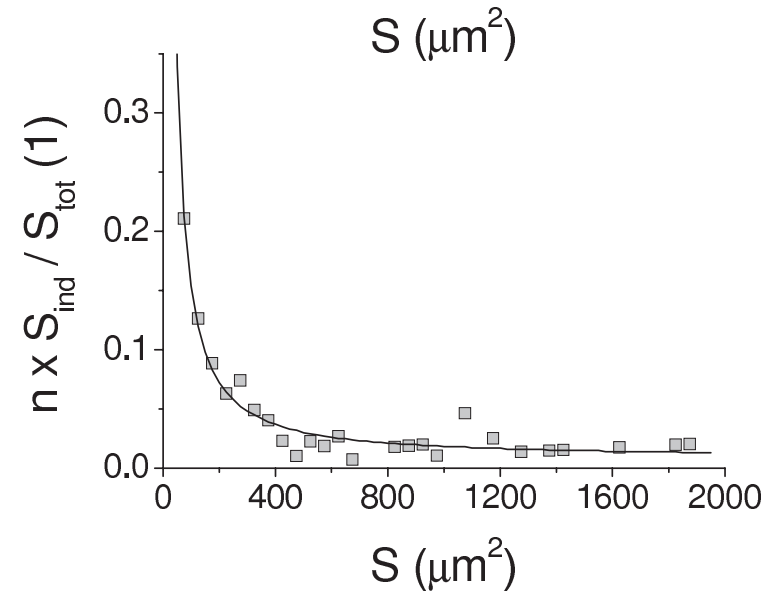

Figure 4. Normalized fragment size distribution of clot degradation fragments for three different flow regimes with perfusion channel plasma velocities: slow flow, $v=0.18 \mathrm{~m} / \mathrm{s}(\mathbf{A})$; medium flow, $v=0.75 \mathrm{~m} / \mathrm{s}(\mathbf{B})$; fast flow, $v=1.5 \mathrm{~m} / \mathrm{s}(\mathbf{C})$. Here $n \times S_{\text {ind }} / S_{\text {tot }}$ denotes a relative proportion of the total area of all fragments with their sizes $S_{\text {ind }}$ between $S-25 \mu \mathrm{m}^{2}$ and $S+25 \mu \mathrm{m}^{2}$ (the bin width is $50 \mu \mathrm{m}^{2}$ ). fibrin network. With faster flow, clot fragments become larger and their total count higher. In the slow flow regime only a few individual RBCs can be seen in the microscopy image (Fig. 3A), in the medium flow regime the fragment count increases to several tens (Fig. 3B), while in the fast flow regime the total count and size of the fragments was further increased (Fig. 3C).

The dependence of the size of fragments and their count on the flow rate was quantitatively analyzed by the fragment tracking software. The results of this analysis are shown in Fig. 4 by a series of graphs that depict the normalized fragment area distributions, i.e., the proportion of different fragment sizes per total area of all fragments in a given flow regime. In the slow flow regime the fragment fraction of the analyzed area (the area of all analyzed fragments within FOV divided by the FOV area) was $0.3 \pm 0.1 \%$. The fraction increased to $3 \pm 1 \%$ in the medium flow regime and to $4 \pm 1 \%$ in the fast flow regime. The differences in fragment fractions were significant between the slow and medium flow regime and between the slow and fast flow regime, while the differences were not significant between the medium and fast flow regime. This result clearly indicates a faster mechanical thrombolysis with a faster flow. As no clusters were found in the slow flow regime, the normalized fragment area distribution had just one peak at the single RBC area (Fig. 4A). In the medium flow regime (Fig. 4B), in addition to single RBCs, clot fragments of $2-10 \mathrm{RBCs}$ were found. The average fragment area in the medium flow regime was $273 \pm 60 \mu \mathrm{m}^{2}$. As evident from the normalized fragment size distribution, the distribution peak was still located at the single RBC area, however, the distribution had a small tail corresponding to clot fragments of more than one $\mathrm{RBC}$. In the fast flow regime, the normalized fragment size distribution was qualitatively similar to the medium flow distribution, but with larger tail that corresponds to larger clot fragments (Fig. 4C). The largest fragments in the fast flow regime consisted of up to $30 \mathrm{RBCs}$ and the average RBC cluster area was 510 $\pm 100 \mu \mathrm{m}^{2}$. With increasing flow velocity the proportion of single RBC fragments decreased and the proportion of larger fragments of more RBCs increased.

Two-tailed Mann-Whitney statistical analysis of measured normalized fragment size distributions showed that differences between the distributions with slow and medium flow and with slow and fast flow are statistically significant $(p<0.05)$, while the difference between the distributions with medium and fast flow is not statistically significant $(p>$ $0.05)$. The best fit parameters of the power-law distribution (Eq. (6)) to the experimentally obtained normalized clot fragment distribution with medium flow regime (Fig. 4B) were equal to $A=0.016 \pm 0.007, B=0.63 \pm 0.04$ and $C=1.61$ $\pm 0.16\left(R^{2}=0.98, \chi_{\text {red }}^{2}=15 \cdot 10^{-5}\right)$, while the corresponding parameters were equal $A=0.009 \pm 0.005, B=0.33 \pm 0.03$ 
and $C=1.19 \pm 0.13\left(R^{2}=0.95, \chi^{2}\right.$ red $\left.=12 \cdot 10^{-5}\right)$ with fast flow regime.

The tail ends of the normalized fragment size distributions in Fig. 4 were used to estimate the maximum fragment areas as a function of flow velocity. The areas were $65 \pm 5 \mu \mathrm{m}^{2}, 950$ $\pm 250 \mu \mathrm{m}^{2}$ and $1850 \pm 250 \mu \mathrm{m}^{2}$ for slow, medium and fast flow, respectively. Square roots of the maximum fragment areas were used to obtain the corresponding linear dimensions (equivalent to the side $a$ of the cubic fragment used in the model), i.e., $a_{\max }=\left(S_{\max }\right)^{1 / 2}$. In Fig. 5 , the linear dimensions of the largest fragments are plotted as a function of the flow velocity. The straight line in Fig. 5 represents the best fit of the model (Eq. 4, laminar flow) to the experimental data. The slope of the line was equal to $f_{L} / k_{V}=32 \cdot 10^{-6} \pm 4 \cdot 10^{-6} \mathrm{~s}$, while the coefficient of determination was equal to $R^{2}=0.94$ and the reduced chi-squared was equal to $\chi^{2}$ red $=4.3$.

\section{Discussion}

This study provides microscopic level analysis of thrombolysis using optical microscopy with the corresponding phenomenological mathematical model that supports our experimental findings, i.e., thrombolysis does not depend only on enzymatic reactions of the fibrinolytic system but also on mechanical forces of streaming blood exerted to the clot surface. Our experimental data confirmed that sizes of the removed clot fragments are dependent on the flow velocity of axially streaming plasma containing the thrombolytic agent rt-PA (Figs. 3-5). In the thrombolytic experiments with whole-blood clots, an 8-fold increase in the velocity of streaming plasma resulted in a 13-fold increase in the fragment fraction of the analyzed area and an even more dramatic 29-fold increase in the area of the largest clot fragment. Fig. 4 reveals that with increasing plasma flow velocity the proportion of single RBCs sloughed from the clot decreased, while the proportion of RBC clusters increased. As RBC clusters are 3-dimensional objects, but fragment tracking software is able to assess only cluster areas, it was difficult to obtain the exact number of RBCs in a cluster. If the clusters were cubic (as in our simplified model assumption) or spherical, the number of RBCs in a cluster would be proportional to the measured area to the power of $3 / 2$. Since the RBC cluster shapes were often elongated, this estimation is only approximate.

The model for the dependence of the maximum clot fragment size on flow velocity in the laminar flow regime (Eq. 4) is in agreement to the measured data in Fig. 5. The model predicts a linear dependence on the size of the clot fragments in the laminar flow regime and a quadratic one in the turbulent flow regime. According to flow parameters in the thrombolytic experiments (flow velocity $1.5 \mathrm{~m} / \mathrm{s}$, diameter of the flow channel $0.7 \mathrm{~mm}$ ), Reynolds number was

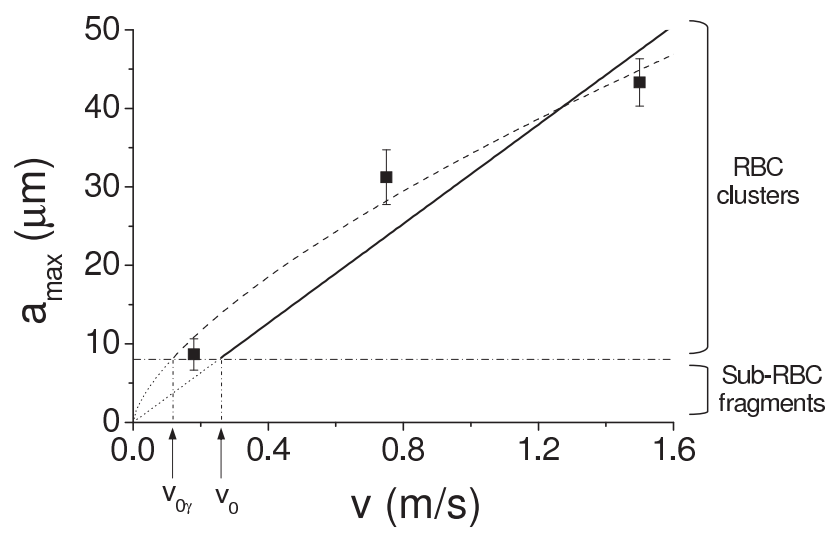

Figure 5. The measured dependence of the maximum clot fragment size (linear dimension of the largest clot fragment, i.e., $a_{\text {max }}=\left(S_{\text {max }}\right)^{1 / 2}$, on the velocity of streaming plasma $v$ (squares). The straight line represents the best fit of the model assuming laminar flow and volume binding forces (volume-binding model: $F_{b}=k_{V} a^{3}$ ) to experimental data, while the dashed line represents the best fit of a modified model, which assumes laminar flow and arbitrary dimensionality of binding forces $\left(\gamma\right.$-model: $\left.F_{b}=k_{\gamma} a^{\gamma}\right)$. The best fit of the $\gamma$-model to experimental data yields $\gamma=3.4 \pm$ 0.2 . Velocities $v_{0}=0.25 \mathrm{~m} / \mathrm{s}$ and $v_{0 \gamma}=0.13 \mathrm{~m} / \mathrm{s}$ denote thresholds for mechanical thrombolysis for the volume-binding model and $\gamma$-model, respectively.

at most $\operatorname{Re}=780$, which is well below the limiting value of 2000 for the persistence of a turbulent flow. Therefore mechanical forces of streaming plasma on the clot surface were of a viscous nature and were thus proportional to the plasma velocity. With the progression of thrombolysis the perfusion channel widens and the flow velocity drops, providing that the volume flow is preserved. In that case Re would decrease during thrombolysis and the laminar flow would never revert into turbulent. However, the initially smooth clot surface can during the progression of thrombolysis gradually turn into a rugged one due to a removal of larger clot fragments. This may lead to a formation of flow niches, which are areas of potential development of turbulences (Leonardi et al. 2004; Chen et al. 2009).

Comparison between the experimental and model data in Fig. 5 indicates that the binding force of clot fragments to the remaining clot was proportional to its volume rather than to its surface. Namely, the model for the dependence of the maximum clot fragment size on the flow velocity in Eq. (4) is based on the assumption that the binding force of the clot fragment is proportional to its volume. If the force were proportional to the surface (the model in Eq. 3), no degradation fragment size dependence on the flow velocity should be obtained, i.e., above the threshold velocity all sizes of degradation fragments should be equally represented, which was not the case in our experiments. 
The proportionality of the clot fragment binding force to its volume could be explained by the 3 -dimensional structure of the fibrin network that forms the scaffold of the clot (Weisel 2007). Clot fragments that are to be removed are not only attached to the rest of the clot along a two-dimensional surface, but also vertically, i.e., 3-dimensionally. This means that deformation of the clot occurs also in the vicinity of the fragment that is to be removed. Thus, the force for fragment removal, in addition to the tearing force that could break the surface bonds of the fragment, must also incorporate a deformation force that would deform the fragment and the clot in its vicinity. The deformations, which are caused by shear forces of streaming plasma, are needed to enable the clot fragment release by pulling or rolling. The tearing force is proportional to the fragment surface, while the deformation force may also depend on other volume-related geometrical elements of the fragment.

The experimentally obtained normalized fragment area distributions showed a decreasing proportion of clot fragments with an increasing clot fragment area (Fig. 4), ranging from the area of a single RBC to the area of the largest clot fragments. Our present theory provides no explanation for the clot fragment distribution profile. This remains a topic for future studies. The theory also assumes a cubic shape of degradation fragments, which is a simplification. In the experiments clot fragments were often elongated and thus akin to block-shapes (Fig. 3). The theoretical model could be improved by incorporating a more generalized shape of degradation fragments. Also, the accuracy of the visible degradation fragment size distributions could be improved if more model blood clots were analyzed. In addition, the proposed theoretical models could be more accurately verified if thrombolysis would be performed at more velocities. However, each of our thrombolytic experiments required a substantial amount of human plasma that was available to us only in limited quantities.

In our experiments flow was laminar so that only laminar flow models are relevant (Fig. 1A,C). Between the two models for laminar flow (surface or volume binding), better agreement with experimental data was obtained when binding forces were assumed volume dependent $\left(F_{b}=k_{V} a^{3}\right)$. The agreement can be further improved if other dimensionality of the binding force than surface or volume is allowed. An example of this is a binding force defined by $F_{b}=k_{\gamma} a^{\gamma}$ ( $\gamma$-model), in which the parameter $\gamma$ corresponds to a dimensionality of the binding force. The $\gamma$-model with laminar flow fits best to experimental data when $\gamma=3.4 \pm 0.2$ and $f_{L} / k_{\gamma}=\left(34.2 \cdot 10^{-6}\right)^{\gamma^{-2}} \mathrm{~s} / \mathrm{m}^{3-\gamma}$ (dashed curve in Fig. 5) with the coefficient of determination equal to $R^{2}=0.99$ and the reduced chi-squared equal to $\chi_{\text {red }}^{2}=1.1$. This result suggests that forces binding RBCs are not only of a shortrange but may also be of a long-range due to viscoelastic properties of the fibrin meshwork (Weisel 2008). The model assuming volume binding forces and laminar flow yields the following velocity threshold for mechanical thrombolysis: $v_{0}=v_{V L}=a_{R B C} k_{V} / f_{L}=0.25 \mathrm{~m} / \mathrm{s}$. A similar threshold velocity can be obtained also for the $\gamma$-model with laminar flow $v_{0 \gamma}=v_{V L}=a_{R B C}{ }^{\gamma-2} k_{\gamma} / f_{L}=0.13 \mathrm{~m} / \mathrm{s}$.

Both of the above considered models assume that forces of streaming plasma have origin in flow resistance and should therefore reduce to zero at no-flow conditions. This has as a consequence an absence of mechanical thrombolysis at zero flow velocity, i.e., both models for the maximum clot fragment size as a function of flow velocity should extrapolate to zero fragment size at zero flow velocity (dotted curves in Fig. 5).

It would be very interesting to extend our observations to the molecular level of fibrin degradation fragments, but that would require the use of purified fibrin clots instead of whole-blood clots and the use of a perfusing fluid containing only components of the fibrinolytic system instead of blood plasma. In whole-blood clots fibrin comprises less than $1 \%$ of the total clot mass, and in our experimental system the clot volume was about $0.2 \mathrm{ml}$ in comparison to the $200 \mathrm{ml}$ of streaming plasma. These proportions explain why plasma proteins completely overshadow the minute quantities of fibrin degradation fragments and make their biochemical detection extremely difficult.

In this work, the novel idea that thrombolysis of nonocclusive blood clots is not just a biochemical process, but strongly depends on mechanical forces of streaming plasma, was confirmed experimentally and theoretically. The optical microscopy analysis of plasma containing removed clot fragments revealed that the sizes of the largest clot fragments during thrombolysis are linearly proportional to the velocity of streaming plasma, which is in agreement with the proposed theoretical model.

Acknowledgements. We thank Dr. Dragoslav Domanovič from the Blood Tranfusion Centre of the Republic of Slovenia for providing human plasma.

\section{References}

Blinc A., Francis C. W. (1996): Transport processes in fibrinolysis and fibrinolytic therapy. Thromb. Haemost. 76, 481-491

Chen Y., C. Zhang C., Shi M., Peterson G. P. (2009): Role of surface roughness characterized by fractal geometry on laminar flow in microchannels. Phys. Rev. E. 80, 026301 http://dx.doi.org/10.1103/PhysRevE.80.026301

Collen D., Lijnen H. R. (1995): Molecular basis of fibrinolysis, as relevant for thrombolytic therapy. Thromb. Haemost. 74, 167-171

Dao M., Lim C. T., Suresh S. (2003): Mechanics of the human red blood cell deformed by optical tweezers. J. Mech. Phys. Solids. 51, 2259-2280 http://dx.doi.org/10.1016/j.jmps.2003.09.019 
Grobelnik B., Vidmar J., Tratar G., Blinc A., Sersa I. (2008): Flowinduced permeation of non-occlusive blood clots: an MRI study and modelling. Eur. Biophys. J. 37, 1229-1233 http://dx.doi.org/10.1007/s00249-008-0342-8

Kadono T. (1997): Fragment mass distribution of platelike objects. Phys. Rev. Lett. 78, 1444-1447 http://dx.doi.org/10.1103/PhysRevLett.78.1444

Kandarpa K. (1999): Catheter-directed thrombolysis of peripheral arterial occlusions and deep vein thrombosis. Thromb. Haemost. 82, 987-997

Kucher N., Goldhaber S. Z. (2006): Risk stratification of acute pulmonary embolism. Semin. Thromb. Hemost. 32, 838-847 http://dx.doi.org/10.1055/s-2006-955466

Landau L. D., Lifshitz E. M. (1995): Fluid Mechanics. ButterworthHeinemann, Oxford

Leonardi S., Orlandi P., Djenidi L., Antonia R. A. (2004): Structure of turbulent channel flow with square bars on one wall. Int. J. Heat Fluid Fl. 25, 384-392 http://dx.doi.org/10.1016/j.ijheatfluidflow.2004.02.022

Marder V. J., Francis C. W. (1983): Plasmin degradation of crosslinked fibrin. Ann. N. Y. Acad. Sci. 408, 397-406 http://dx.doi.org/10.1111/j.1749-6632.1983.tb23260.x

Sakharov D. V., Rijken D. C. (2000): The effect of flow on lysis of plasma clots in a plasma environment. Thromb. Haemost. 83, 469-74

Sersa I., Vidmar J., Grobelnik B., Mikac U., Tratar G., Blinc A. (2007): Modelling the effect of laminar axially directed blood flow on the dissolution of non-occlusive blood clots. Phys. Med. Biol. 52, 2969-2985

http://dx.doi.org/10.1088/0031-9155/52/11/003
Šabovič M., Lijnen H. R., Keber K., Collen D. (1990): Correlation between progressive adsorption of plasminogen to blood clots and their sensitivity to lysis. Thromb. Haemost. 64, 450-454

Timar G., Blomer J., Kun F., Herrmann H. J. (2010): New Universality Class for the Fragmentation of Plastic Materials. Phys. Rev. Lett. 104, 095502 http://dx.doi.org/10.1103/PhysRevLett.104.095502

Tratar G., Blinc A., Strukelj M., Mikac U., Sersa I. (2004): Turbulent axially directed flow of plasma containing rt-PA promotes thrombolysis of non-occlusive whole blood clots in vitro. Thromb. Haemost. 91, 487-496

Weisel J. W. (2007): Structure of fibrin: impact on clot stability. J. Thromb. Haemost. 5 (Suppl. 1), 116-124 http://dx.doi.org/10.1111/j.1538-7836.2007.02504.x

Weisel J. W. (2008): Biophysics. Enigmas of blood clot elasticity. Science. 320, 456-457 http://dx.doi.org/10.1126/science. 1154210

White-Bateman S. R., Schumacher H. C., Sacco R. L., Appelbaum P. S. (2007): Consent for intravenous thrombolysis in acute stroke: review and future directions. Arch. Neurol. 64, 785-792 http://dx.doi.org/10.1001/archneur.64.6.785

Zidanšek A., Blinc A., Lahajnar G., Keber D., Blinc R. (1995): Finger-like lysing patterns of blood clots. Biophys. J. 69, 803-809

http://dx.doi.org/10.1016/S0006-3495(95)79954-4

Received: September 9, 2011

Final version accepted: February 17, 2012 